\title{
ASO Author Reflections: Geographic Disparities in Referral and Oncologic Outcomes in Intrahepatic Cholangiocarcinoma
}

\author{
Thomas L. Sutton, MD ${ }^{1}$, and Skye C. Mayo, MD, MPH, FACS, FSSO ${ }^{2}$ \\ ${ }^{1}$ Department of Surgery, Oregon Health and Science University (OHSU), Portland, OR; ${ }^{2}$ Department of Surgery, Division \\ of Surgical Oncology, The Knight Cancer Institute at OHSU, Oregon Health and Science University (OHSU), Portland, OR
}

\section{PAST}

Patients with malignancies that are rare and aggressive, such as intrahepatic cholangiocarcinoma (ICC), benefit from the clinical experience and spectrum of treatments available at high-volume referral centers. ${ }^{1}$ In pancreatic cancer, for example, such high-volume centers have been associated with improved outcomes for patients despite more advanced disease in the patient population that is treated. ${ }^{2}$ This phenomenon extends to patients with metastatic disease receiving palliative chemotherapy., National Cancer Institute (NCI)-designated Comprehensive Cancer Centers represent the epitome of referral centers for cancer in the US, offering cutting-edge cancer care, including investigator-initiated clinical trials and other investigative efforts. Unfortunately, the geographic distribution of NCI Cancer Centers is heavily skewed to the Eastern United States, and patients in the Western United States must often travel long distances for access to programs offering comprehensive cancer care. For example, Alaska, North Dakota, South Dakota, Montana, Idaho, Iowa, and Nevada do not have NCI Cancer Centers. Historically, no data on the impact of such geographic disparities on treatment and outcomes in ICC were available. Similarly, the proper avenues to address geographic disparities in cancer care have been elusive, as evaluation

(C) Society of Surgical Oncology 2021

First Received: 12 July 2021

Accepted: 14 July 2021;

Published Online: 17 August 2021

S. C. Mayo, MD, MPH, FACS, FSSO

e-mail: mayos@ohsu.edu and treatment at a referral center would often require a prohibitive amount of time off work for patients in remote locations.

\section{PRESENT}

In this issue, we report an analysis of patients with ICC diagnosed within the predominately rural state of Oregon. ${ }^{5}$ We utilized the Oregon State Cancer Registry to identify treatment patterns for patients with ICC, identifying three groups: those diagnosed and treated at referral centers, those diagnosed and treated at community centers, and those with parts of first-course therapy taking place in both settings. Referral centers were defined as programs offering multidisciplinary oncology care and clinical trials for ICC. We showed that patients treated in the combined community center plus referral center settings had improved survival compared with patients treated only at referral centers, and both of which had improved survival compared with patients who were treated only in the community without any referral center involvement. Additionally, referral center involvement in first-course treatment was associated with higher odds of receiving a curative-intent resection, radiation, and systemic chemotherapy, which were each associated with improved survival. Surprisingly, metastatic disease at diagnosis was independently associated with lower odds of referral center treatment. Finally, farther distance to the nearest referral center was independently associated with lower odds of referral center treatment. These findings are useful in characterizing the disparities in referral and treatment that exist in states with a predominately rural population, and are likely reflective of the difference in survival outcomes that may result. These disparities may be particularly 
pronounced for malignancies such as ICC, where novel treatments such as targeted therapeutics may be more available or appropriately utilized at referral centers.

\section{FUTURE}

It is incumbent upon clinicians at NCI Comprehensive Cancer Centers and other referral centers with treatment programs for rare and aggressive malignancies such as ICC to ensure that all patients within their catchment basin have access to high-quality cancer care. Prior to the severe acute respiratory syndrome coronavirus 2 (SARS-CoV-2) pandemic, long distances often stymied outreach attempts to remote communities, and telemedicine technology was not widely available or utilized by oncologists. However, lessons learned can and should be leveraged to improve the oncologic care for patients in remote locations. Telemedicine can eliminate hours of travel across hundreds of miles for an initial evaluation, and what previously required patients to miss a day or more of work can now be accomplished during a lunch break. Even for patients who do not have a sufficient performance status to undergo a curative-intent resection, such as the major hepatectomies often required for ICC, telemedicine offers the option for referral centers to guide the care administered locally, even if that care is predominantly palliative in nature.

As increasing emphasis is placed on centralization of oncologic care due to the benefits of treatment at highvolume referral centers, the medical community is at risk of potentially overlooking patients in remote locations. If recent lessons on delivering care remotely are leveraged moving forward, the SARS-CoV-2 pandemic can be a watershed moment for oncologic care of patients in rural areas worldwide. As evidence shows, treatment at highvolume referral centers has measurable benefits, not just in the surgical population but even in patients with metastatic disease. We therefore encourage the continued use and expansion of telemedicine in the post-pandemic era to facilitate rapid evaluation and treatment planning for patients with cancer, particularly for malignancies where cutting-edge therapies promise the greatest impact to oncologic outcomes when guided by the clinical experience at cancer centers.

FUNDING This manuscript did not receive any specific grant from funding agencies in the public, commercial, or not-for-profit sectors.

DISCLOSURES Thomas L. Sutton and Skye C. Mayo have no conflicts of interest or financial ties to disclose.

\section{REFERENCES}

1. Wu L, Tsilimigras DI, Paredes AZ, et al. Trends in the incidence, treatment and outcomes of patients with intrahepatic cholangiocarcinoma in the USA: facility type is associated with margin status, use of lymphadenectomy and overall survival. World $J$ Surg. 2019;43(7):1777-87.

2. Lidsky ME, Sun Z, Nussbaum DP, Adam MA, Speicher PJ, Blazer DG 3rd. Going the extra mile: improved survival for pancreatic cancer patients traveling to high-volume centers. Ann Surg. 2017;266(2):333-8.

3. Haj Mohammad N, Bernards N, Besselink MG, et al. Volume matters in the systemic treatment of metastatic pancreatic cancer: a population-based study in the Netherlands. J Cancer Res Clin Oncol. 2016;142(6):1353-60.

4. Haj Mohammad N, Bernards N, van Putten M, Lemmens V, van Oijen MGH, van Laarhoven HWM. Volume-outcome relation in palliative systemic treatment of metastatic oesophagogastric cancer. Eur J Cancer. 2017;78:28-36.

5. Sutton TL, Walker BS, Nabavizadeh N, et al. Geographic disparities in referral and oncologic outcomes in intrahepatic cholangiocarcinoma: a population-based study. Ann Surg Oncol. 2021. https://doi.org/10.1245/s10434-021-10650-6.

Publisher's Note Springer Nature remains neutral with regard to jurisdictional claims in published maps and institutional affiliations. 